\title{
El Castillo de La Atalaya (siglo XVIII), en Cartagena (Región de Murcia, España). Análisis formal y constructivo
}

The Atalaya Castle (eighteenth century), in Cartagena (Region of Murcia, Spain). Formal and constructive analysis

\author{
Federica Carta $^{\text {a }}$, Diego Ros McDonnell ${ }^{\text {b }}$, Pedro Enrique Collado Espejo ${ }^{\text {c }}$ \\ Universidad Politécnica de Cartagena, Cartagena, Spain

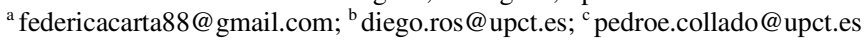

\begin{abstract}
The Atalaya Castle (eighteenth century) is one of the military fortifications that were part of the defense of Cartagena. The defensive system of the period was composed of an important walled enclosure, which surrounded the city, the arsenal, and a group of fortresses outside the city wall, located on the nearby hills. One of these defensive constructions is the Atalaya Castle or Fort, located to the west of the city from its position it protected the population from attacks both by land and by sea. To the north and west by land, through the Almarjal and the Pelayo mountains, the south by sea covered the possible landings in the bays of the Algameca Grande and the Algameca Chica. The building is a magnificently construction, the fort has a pentagon ground plan with five bastions at each angle. It has an interior building in $U$ arranged on a solid bastioned platform the whole complex is surrounded by a dry moat. The fortification present certain formal elements used in other constructions that had been lifted in the city at that time, circumstance gave unity to the whole. The materials consisted of employed mainly stone and brick, the constructive elements introduce certain heterogeneity. The purpose of the communication is to present the results of the comprehensive analysis carried out in the Atalaya Castle as well as to contribute, through its dissemination to raise awareness of the need for its restoration and enhancement. Research has studied the characteristics of the formal and constructive system of the fortification currently in a state of semiabandonment, a proposal has also been conducted for a new cultural use as a guarantee of its correct recovery and conservation.
\end{abstract}

Keywords: Atalaya Castle, Cartagena, military architecture, bastioned fortification.

\section{Introducción}

El Castillo de La Atalaya es una de las fortificaciones militares que formaban parte de la defensa de Cartagena levantadas durante el siglo XVIII. Este texto, consecuencia del Trabajo Fin de Master, con título "El Castillo de La Atalaya, Estado y Sistema Constructivo" (realizado por Federica Carta, bajo la dirección académica de Diego Ros McDonnell y Pedro E. Collado Espejo), tiene por objeto mostrar el estudio realizado de la fortaleza, reuniendo y ampliando la información existente sobre la misma, además de evidenciar la importacia de las contrucciones militares levantadas en aquella época en el municipio de Cartagena. Así mismo, durante la elaboración del trabajo se procedió a documentar gráficamente el gran patrimonio arquitectónico militar del siglo XVIII de Cartagena, aportando una visión analítica del rol que desempeñó el Casti- 
llo y mostrando la importancia de la construcción militar como referente patrimonial así como su relevancia social y cultural para su ciudad.

El Castillo de La Atalaya, como las restantes fortificaciones de Cartagena del siglo XVIII, está declarado Bien de Interés Cultural con carác- ter de Monumento, según la Disposición Adicional Segunda de la Ley 16/1985, de 25 de junio, del Patrimonio Histórico Español. Así mismo, el vigente Plan General Municipal de Ordenación de Cartagena, le asigna el máximo grado de protección, circunstancias que no han evitado su progresivo deterioro.

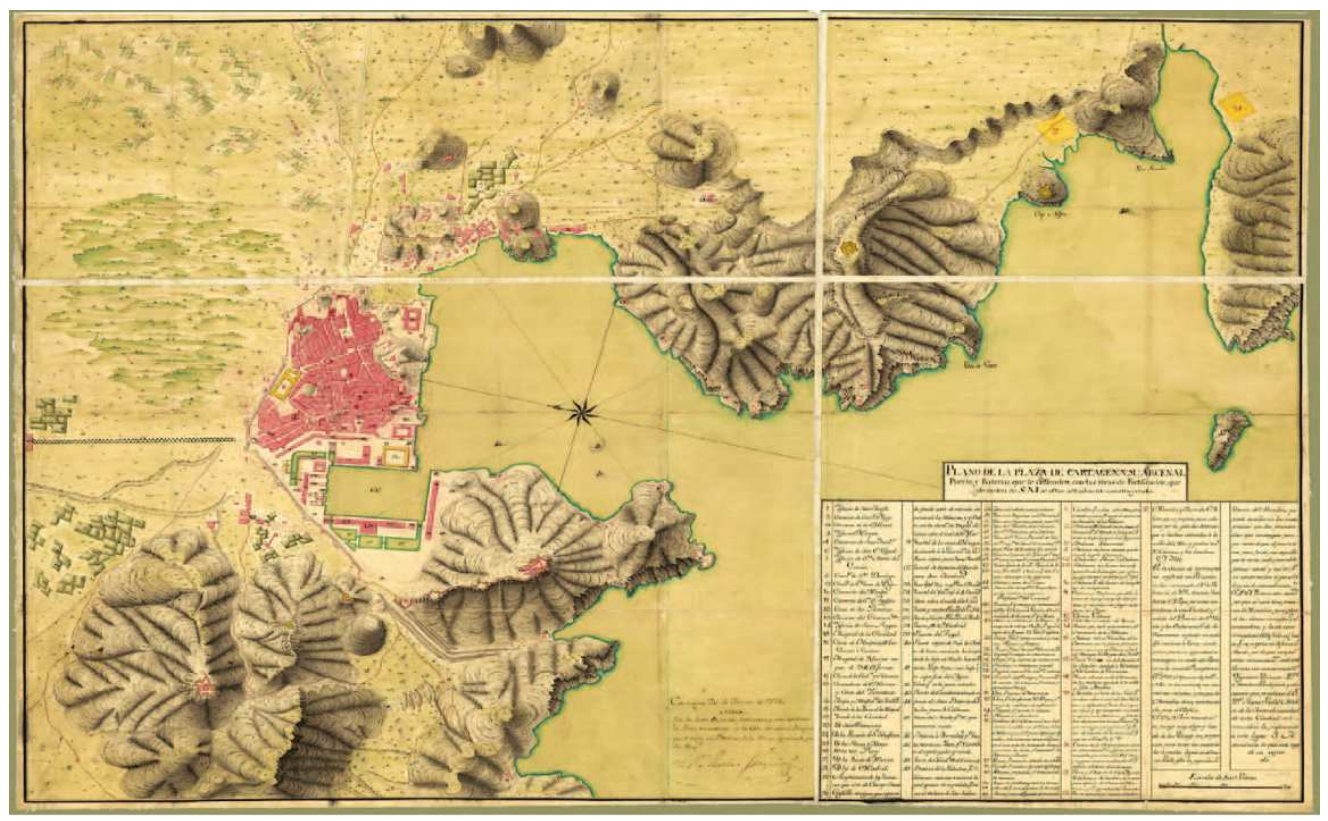

Fig. 1. "Plano de la Plaza de Cartagena, su Arsenal, Puerto y Baterías que le defienden: con las obras de fortificación, que de orden de S.M. se están actualmente construyendo". Ministerio de Defensa, Archivo Cartográfico y de Estudios Geográficos del Centro Geográfico del Ejército. Mateo Vodopich, 1778 (Martínez López, 2015).

A partir de finales del XVI, la Corona comienza la construcción de numerosas torres costeras para la defensa del litoral murciano y el Concejo de Cartagena, por falta de recursos económicos, se centrará en la reparación de la muralla y del Castillo de La Concepción, En general fueron construcciones que, en el mejor de los casos, sólo podían hacer frente a un ataque de pequeña envergadura, por ello no se puede afirmar que el estado de la defensa fuera del todo satisfactorio (Gómez Vizcaíno, Munuera Navarro, 2002a).

El desarrollo de la artillería durante el siglo XV había convertido en obsoletas las construcciones defensivas realizadas hasta entonces. Los castillos y murallas ya no podían resistir la capacidad destructiva de los nuevos proyectiles. Por ello, los ingenieros militares idearon las fortificaciones abaluartadas, unas construcciones con muros de menor altura y caras exteriores inclinadas y de mayor espesor. Estas nuevas construcciones permitían disponer de artillería tras los merlones y garantizaban el máximo control de las zonas exteriores de las fortalezas.

A partir de entonces, Cartagena se convirtió en uno de los enclaves militares más importantes en la defensa de la costa española del Mediterráneo. A finales del siglo XVI la ciudad comienza a renacer. La designación del puerto de Cartagena como apostadero de la flota de Galeras impulsó su desarrollo social y económico, aunque el momento importante de la ciudad llegaría posteriormente. 
En los siglos XVI y XVII la ciudad tuvo un gran aumento demográfico, que encontró su punto máximo entre mitad y finales del siglo siguiente, circunstancia que provocó una gran expansión urbana fuera del recinto fortificado. Cartagena estaba poniendo las bases para volver a ser una de las ciudades más importantes de España.

\section{Circunstancias de Cartagena en los siglos XVII y XVIII}

Los principales avances de la arquitectura defensiva del siglo XVIII tuvieron origen francés, especialmente gracias al Mariscal Vauban principal ingeniero militar de su tiempo. La llegada de los Borbones a la corona de España impulsó la introducción de las nuevas tipologías defensivas que, en Cartagena, culminan bajo el reinado de Carlos III (Gómez Vizcaíno, Munuera Navarro, 2002b).

La instauración de la dinastía borbónica, tras la Guerra de Sucesión (1701-1713), causó la pérdida del Reino de Nápoles para la Corona española y la decisión de establecer la base naval del Mediterráneo en Cartagena, aprovechando su estratégica ubicación geográfica y en la bondad de su puerto. Con ello, comienzan a llegar los primeros ingenieros ilustrados para el estudio y mejora de la defensa de la ciudad y, con el desarrollo de estos proyectos, se cambiará la configuración urbana y del entorno. La creación de los Departamentos Marítimos por la Real Orden de 5 de diciembre de 1726 supone el comienzo de una intensa actividad de los ingenieros militares, que se prolongará casi ochenta años, elaborando gran cantidad de proyectos. La Corona crea tres Departamentos Marítimos: Norte, Sur y Levante, con capitanía en El Ferrol, Cádiz y Cartagena respectivamente. Cada una de ellas debía de tener un arsenal y astilleros, con lo que Cartagena debía tener una fortificación adecuada a su nueva categoría (Gómez Vizcaíno, Munuera Navarro, 2002b).

A principios del XVIII, la monarquía española, con el fin de proteger su presencia en el Mediterráneo, planifica y financia las obras militares en Cartagena, propiciando el auge de la ciudad en todos los ámbitos, y convirtiéndola en plaza fuerte de la Corona (Marzal, 1993). La ciudad dependía totalmente de las inversiones estatales. El refuerzo de las murallas y la construcción del arsenal y cuarteles militares, así como la presencia de la flota en el puerto, trajeron consigo gran cantidad de soldados, trabajadores, comerciantes, marineros, etc., que vivían directa o indirectamente del Estado.

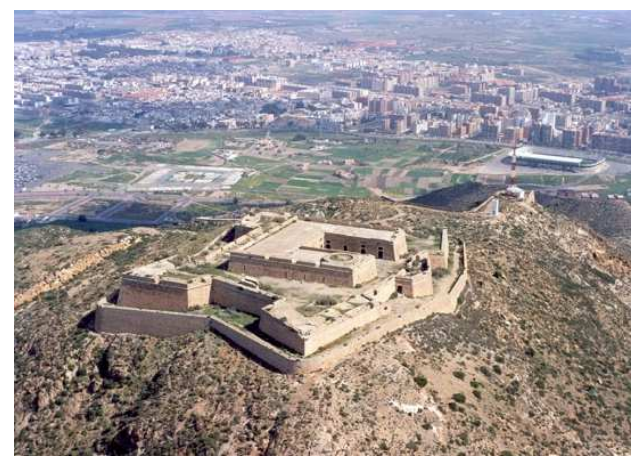

Fig. 2. Vista general aérea del Monte de la Atalaya y del Castillo de la Atalaya, y al fondo Cartagena (http://listarojapatrimonio.org/ficha/castillo-de-laatalaya/).

Cada uno de los elementos del sistema defensivo de la ciudad tenía un objetivo específico, Las murallas y el arsenal protegían el casco urbano, mientras que los fuertes exteriores protegían la población frente al avance de fuerzas atacantes y asedios por tierra (castillos de La Atalaya y Los Moros), y por mar (Castillo de Galeras, y Fuerte Navidad, principalmente). La posición, solidez de la construcción y campo visual (que se extendía hacia la llanura interior), convertían el Castillo de La Atalaya en un elemento determinante de la seguridad y defensa de la ciudad. La sublevación Cantonal de 1873 corrobora esta circunstancia, pues la conquista de esta fortaleza precipitó la capitulación de la ciudad un año después.

A finales del siglo XIX, se puede considerar que Cartagena estaba adecuadamente defendida por su recinto amurallado y las tres fortalezas principales: Galeras, La Atalaya y Los Moros.

Durante el siglo XIX, el ejército prusiano desarrolló unos avances bélicos que marcan el comienzo de la decadencia de las construcciones abaluartadas. La ineficacia se acentuó con los cañones estriados y los obuses cilindro-ojivales, 
gracias a los cuales la artillería incrementó el alcance, la precisión de tiro y la potencia destructiva. Comienza así el abandono de las fortificaciones abaluartadas y el lento proceso de deterioro que ha llevado al precario estado actual de estas edificaciones, si no a la ruina.

Además del desuso, cabe citar como causa de abandono de estas construcciones militares el alto coste de su mantenimiento $\mathrm{y}$, sobre todo, la desidia de las administraciones públicas, especialmente la nacional (propietaria de estas construcciones) y la local.

\section{Emplazamiento}

El Castillo de La Atalaya, localizado al oeste de Cartagena, está emplazado en la cima del monte que le da nombre y que forma parte de la Sierra de Pelayo. La fortaleza, situada a una altura de $242 \mathrm{~m}$ y en un entorno cuya orografía se caracteriza por escarpadas pendientes donde abundan barrancos, domina la ciudad, el arsenal, el barrio extramuros de La Concepción, el Almarjal y la llanura al norte. En esa zona norte de la ciudad se desarrollará, a principios del XX, el Ensanche, y en el sur las ensenadas de la Algameca Grande y de la Algameca Chica. El actual acceso, que parte del barrio de La Concepción, es un camino estrecho (un ancho de unos $3 \mathrm{~m}$ ), firme irregular y una pendiente media del 15\% (Martínez e Iniesta, 2002).

\section{Desarrollo de la construcción}

Según los informes mensuales de obras, la construcción del Castillo comenzó en marzo de 1773 y terminó en junio de 1778 . Gracias a esos informes se ha comprobado la pérdida de algunos, como las bóvedas de media naranja de la garita que cubría la escalera de caracol que permitía el acceso a la azotea y la de la garita que cubría la bajada al aljibe.

Analizando la cartografía histórica (los primeros proyectos de Pedro Martín Zermeño, de 1766, y de Francisco Llobet, de 1773), se aprecia que el diseño de la fortaleza se corresponde con un edificio en $U$ abierta al sur sobre una plataforma maciza de planta trapezoidal con cuatro baluartes en sus vértices. Así mismo los proyectos prevén respectivamente tres y dos revellines, res- pectivamente, que finalmente no se ejecutaron. El proyecto de Llobet sigue fielmente el de Zermeño, si bien potencia la plataforma maciza inferior (aumentando su altura) y mejora su cierre al sur.

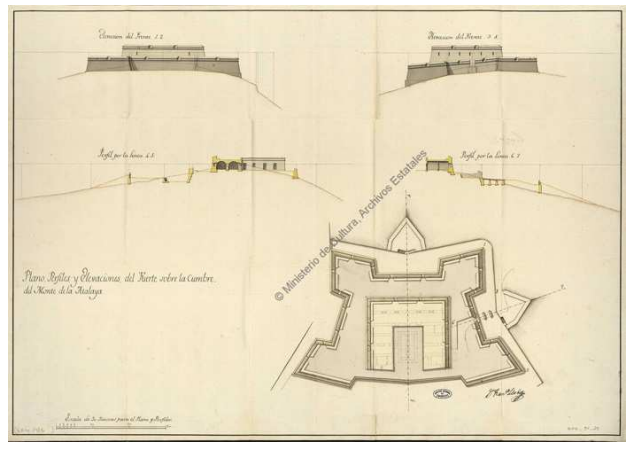

Fig. 3. Plano del proyecto de Francisco Llobet (Ministerio de Cultura, Archivos Estatales).

En 1799, el ingeniero militar Juan José Ordovás dibujó las defensas del Reino de Murcia, y entre éstas se encontraba el Castillo de La Atalaya según quedó terminado bajo la dirección del ingeniero militar Mateo Vodopich, en 1778.

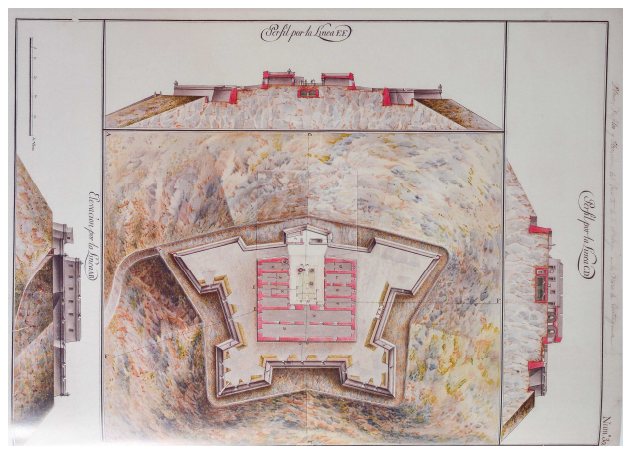

Fig. 4. Plano $\mathrm{n}^{\circ} 30$ (perfiles, plano y alzado del fuerte de La Atalaya). Copiado por Manuel Martínez Nubla (1819), del original de Juan José Ordovás (1799). (Martínez y Munuera, 2005).

Confrontando los distintos planos históricos se puede comprobar la disposición de un quinto baluarte al sur que protege el acceso a la fortaleza, la construcción total del foso seco perimetral y la reducción del número de brocales del aljibe que pasa de tres a dos. Por otra parte, a lo largo de la fase de levantamiento y toma de datos in situ del 
castillo para realizar el TFM y la restitución de los planos actuales, se comprobó un pequeño error en el plano de Ordovás, la escalera de caracol está grafiada en el lugar de una ventana en muro en talud.
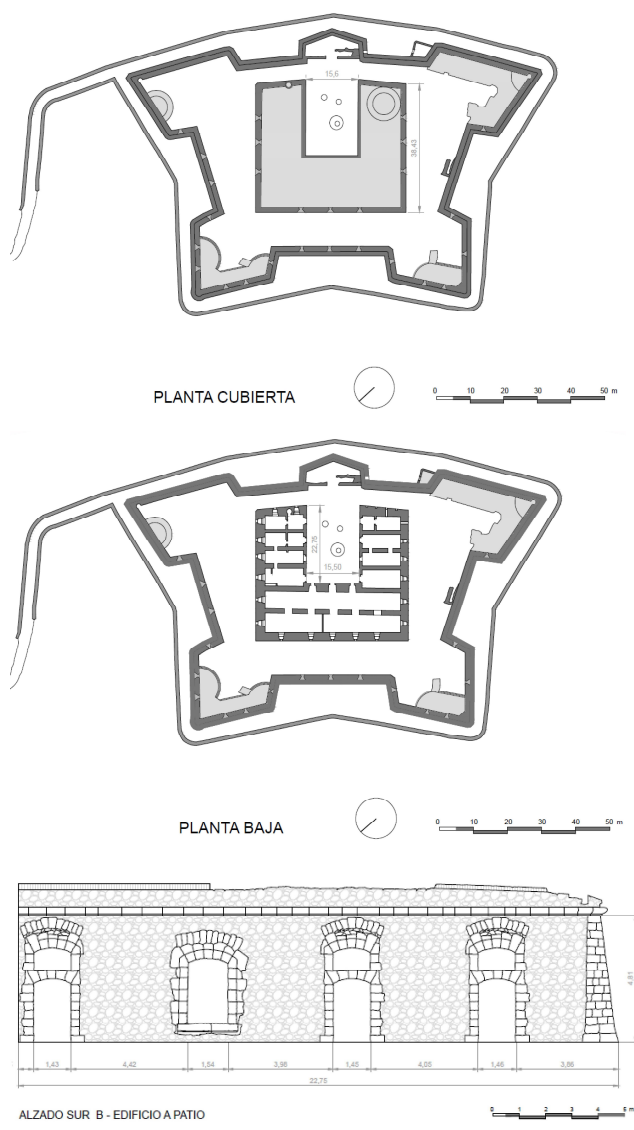

Fig. 5. Planos de cubierta, planta baja y alzado sur del edificio del patio, incluidos en el TFM desarrollado (Federica Carta, 2017).

\section{Sistema constructivo}

El sistema constructivo del fuerte es de muro de carga de mampostería careada con esquinas y brencas de huecos resueltas con sillarejo labrado, con un amplio foso seco perimetral; el acceso está ubicado a sur, resuelto mediante una puerta en recodo en la cara oeste del baluarte más pequeño dispuesto en el centro del frente meridional (Rubio Paredes, 1997). Presenta dos niveles, el nivel inferior es una plataforma maci- za y de forma sensiblemente pentagonal con baluartes en cada vértice. Tanto los baluartes como los lados del polígono interior se unen a través de cortinas o lienzos rectos de muralla.

La puerta principal de acceso al recinto presenta un arco rebajado por un frente y por el otro un arco carpanel. Este tipo de acceso, ampliamente empleado por su gran eficacia desde el punto de vista militar, durante un posible asalto obligaba el enemigo a avanzar con el flanco izquierdo descubierto hacia los defensores (Torres Balbás, 1960). Una vez franqueada esta puerta, había un puente levadizo (actualmente desaparecido), que cortaba las escaleras de acceso al patio de armas del nivel superior o primera planta (Martínez e Iniesta, 2002).
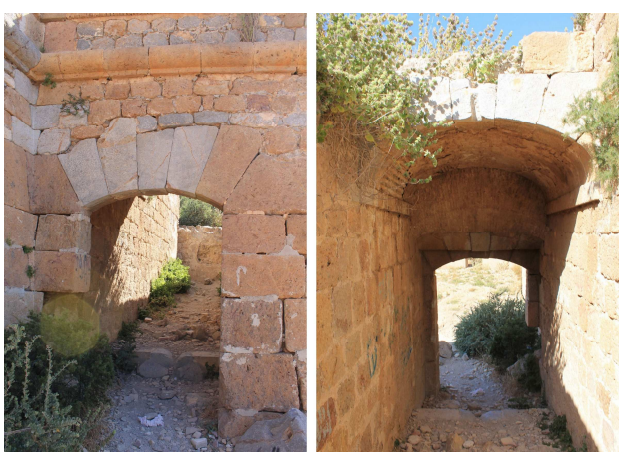

Fig. 6. Acceso a la Fortaleza de la Atalaya (Federica Carta, 2017).

En la planta superior de la fortaleza está ubicado el edificio en forma de $U$ abierta hacia el sur, la cual disponía de varias dependencias. Una de las estancias cuenta con un volumen que combina ladrillo y piedra. Las fachadas que dan al patio presentan aperturas (puertas y ventanas), con arcos escarzanos. Las fachadas exteriores presentan ventanas en muros en talud. Los arcos en talud son poco frecuentes en la práctica (Calvo López, 2007). De hecho, en el Castillo de La Atalaya se alternaron muros de piedra con arcos y bóvedas compuestos por ladrillos.

En la primera sala del ala Este había una escalera de caracol para el acceso a la cubierta, que contaba con parapeto perimetral. Hoy en día la escalera prácticamente ha desaparecido, sólo quedan el primer peldaño y los mechinales de 
los demás en el muro circular de ladrillo. El estudio formal de la única pieza que permanece en su lugar, comparándola con otros ejemplos de la cantería española, indica que la escalera era del tipo de husillo. Se puede ver perfectamente que el arranque de la escalera estaba constituido por una única pieza que comprendía peldaño y poste central. El empleo de esta categoría de escalera se remonta al siglo XIII cuando sirvió para dar respuestas a las intenciones de la arquitectura gótica, aunque fue empleada por algún arquitecto griego en el V siglo a.C.; como en el Templo A de Selinunte, en Castelvetrano, Sicilia, Italia (Sanjurjo Álvarez, 2016).
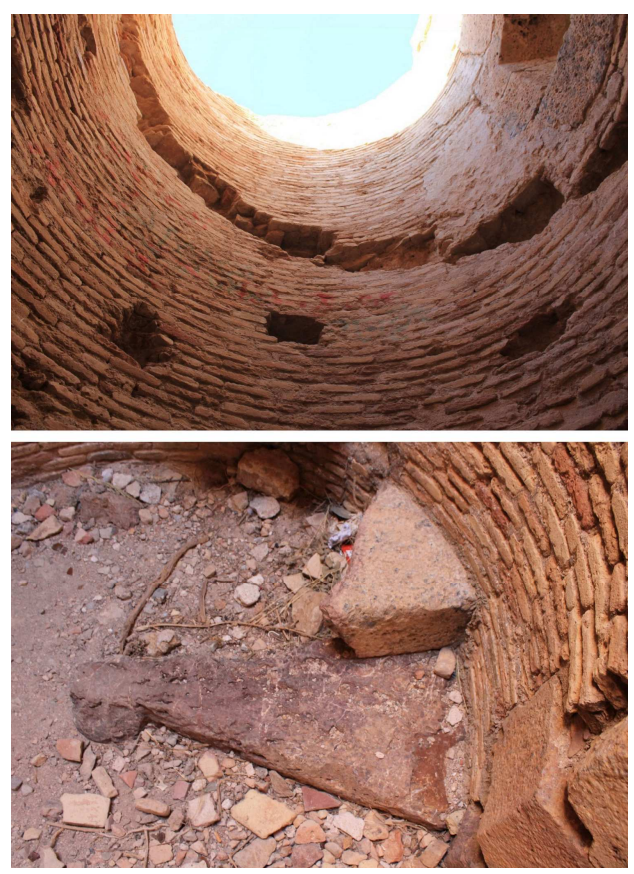

Fig. 7. Detalles de la escalera del Castillo de La Atalaya (Federica Carta, 2017).

El material dominante de los baluartes era la mampostería careada tomada con mortero de cal con contrafuertes y relleno de tierra apisonada con el fin de poder absorber el impacto de la artillería y minimizar su efecto. En cuanto a su ejecución, se conformaba primero el muro de mampostería con contrafuertes y después se rellenaba el interior de tierra. Las esquinas se reforzaban con sillares pétreos que eran tallados convenientemente.
Las estancias del edificio en $\mathrm{U}$ presentan muros de mampostería hasta el arranque de las bóvedas. Estos muros están rematados por una hilada de ladrillo macizo colocado a sardinel para asiento de la propia bóveda. Las aperturas de huecos, en su parte interior, están resueltas bien con brencas de sillarejo, bien con brencas de fábrica de ladrillo macizo. Las cubiertas disponen de bóvedas de cañón realizadas en ladrillo macizo "a prueba de bomba". Este tipo de cubierta estaba preparada para resistir los posibles impactos de proyectiles gracias a una capa de tierra batida colocada, entre la bóveda de ladrillo y el pavimento de la azotea, que absorbía la onda expansiva de las balas de cañón.

\section{Estudio de uso}

Desde el año 2016, el Castillo de La Atalaya es propiedad del Ayuntamiento de Cartagena. A día de hoy, el consistorio no se ha pronunciado de manera contundente acerca de sus intenciones sobre el futuro de la fortaleza, aunque su posible rehabilitación y adecuación para otros usos es un tema recurrente.

Tras las numerosas visitas y con el desarrollo del estudio realizado, nos parece evidente que la fortaleza de La Atalaya no es apta para albergar un uso de tipo cultural (como se ha defendido en numerosos ámbitos sociales y culturales), mientras no se acondicione convenientemente el camino de acceso. Un aspecto que llama mucho la atención es el carácter deportivo que el castillo y el monte han asumido en los últimos años. La actividad más conocida es la "Ruta de las Fortalezas", uno de los mayores eventos deportivos de la Región de Murcia, en la cual participan miles de personas. Este evento incluye el Castillo de La Atalaya como elemento destacado. Durante el camino de subida se pueden apreciar la Cueva de La Atalaya, los túneles militares, un vértice geodésico, un antiguo aljibe, etc., y disfrutar de vistas espectaculares hacia el puerto y la ciudad. Los días despejados, mirando hacia el sureste, es posible ver el Mar Menor, distante unos treinta kilómetros, algo que permite comprender el importante papel de la instalación militar de La Atalaya en la defensa de la costa. 
Así mismo, durante las visitas, nos hemos encontrado con frecuencia a personas realizando escalada libre en cualquiera de las fachadas del edificio de la plataforma superior. Con el abandono del uso militar, esta práctica deportiva, junto con la gran presencia de senderistas que culminan la ascensión al monte, le ha dado una nueva utilidad a la fortaleza. Descartando otras posibles opciones de uso debido a la dificultad de acceso, cabe tomar como referencia el Castle Climbing Centre en Londres, castillo adaptado como gimnasio de escalada y que organiza cursos de escalada, eventos y competiciones a nivel internacional. Por ello, el TFM plantea la rehabilitación arquitectónica integral del Castillo de La Atalaya (incluyendo la recomposición volumétrica de los volúmenes y elementos perdidos), la adecuación del camino de acceso actual (pero con una senda con pendientes más suaves de los actuales y barandillas de madera, para hacerla más segura) y su adecuación para un uso cultural y deportivo; pero siempre desde el respeto a los valores patrimoniales (históricos, arquitectónicos, militares, sociales y culturales) que atesora este monumento. En este sentido, entendemos que es primordial el uso de sistemas constructivos y materiales tradicionales y propios del edificio en cuestión.

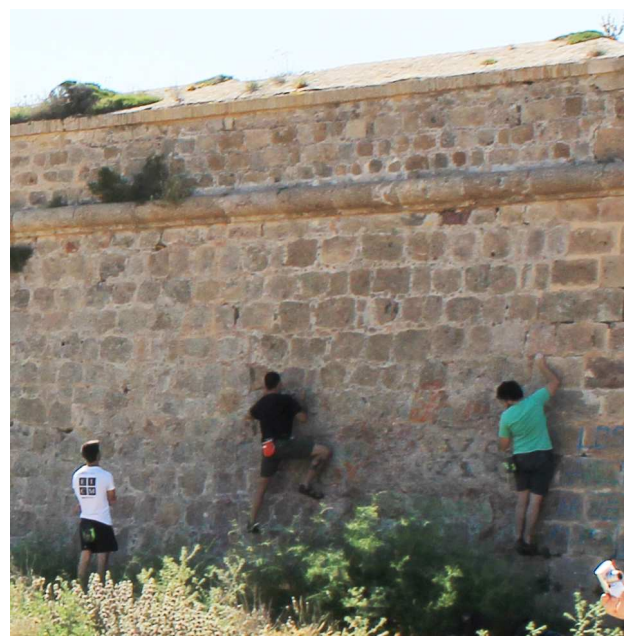

Fig. 8. Práctica de escalada libre en el Castillo de La Atalaya (Federica Carta, 2017).

\section{Conclusiones}

El Castillo de La Atalaya, en Cartagena, a pesar de tener la máxima categoría de protección (BIC con categoría de monumento), se encuentra en la actualidad en un estado de conservación muy próximo al abandono y no se han realizado labores efectivas para su conservación. Desde el año 2016, esta singular construcción militar del siglo XVIII ha pasado a ser propiedad del Ayuntamiento de Cartagena. Sin embargo, en los últimos años no se han adoptado medidas de protección y conservación (el acceso a todo el recinto es libre), por lo que se han perdido muchas piezas importantes del castillo.

Las construcciones militares del siglo XVIII marcan un periodo histórico trascendental para Cartagena que, después de una época oscura (Guerra de Sucesión), renace al convertirse la ciudad en uno de los tres Departamentos Marítimos de España. Así mismo, la importancia de estas construcciones militares se encuentra en el desarrollo urbano de la ciudad y su entorno, siendo un referente patrimonial y cultural tanto para la población como para visitantes.

Las trazas de la fortaleza de La Atalaya, elaboradas por el ingeniero militar Pedro Martín Zermeño en 1766, se mantienen tanto en el proyecto posterior (Francisco Llobet, en 1773), como en la construcción, terminada en 1778 bajo la dirección del también ingeniero militar Mateo Vodopich. Con el estudio histórico-constructivo y el levantamiento planimétrico realizado se ha constatado que solo se han efectuado pequeñas y puntuales modificaciones.

Las dificultades actuales para el acceso seguro hasta el momento así como la presencia habitual de senderistas, corredores y aficionados a la escalada, nos ha llevado a considerar que un nuevo uso posible de la edificación, una vez sea convenientemente rehabilitada, podría ser el cultural y deportivo; pero siempre bajo la premisa del máximo respeto y valoración del bien patrimonial y el entorno natural y paisajístico en el que se encuentra.

Consideramos que el Castillo de La Atalaya aún puede jugar un papel importante en la vida social y cultural de la ciudad. La mejora de accesos y 
la rehabilitación del castillo, con actuaciones similares en el resto de fortificaciones del siglo XVIII, deben ser una prioridad en los próximos años. Con este objetivo se ha desarrollado el
TFM. En este sentido, el trabajo supone un primer paso para sensibilizar a la población, en general, y a las administraciones, en particular.

\section{Bibliography}

Calvo López, J. (2007). "Piezas singulares de cantería en la ingeniería y la arquitectura militar de Cartagena en el siglo XVIII", in Actas del Quinto Congreso Nacional de Historia de la Construcción, instituto Juan de Herrera Ed., Madrid; pp. 166-176.

Carta, F. (2019). "Arquitectura militar del siglo XVIII. El Castillo de la Atalaya en Cartagena. Análisis formal y constructivo. Estudio patológico y de uso", in XXV Jornadas de Patrimonio Cultural de la Región de Murcia, Consejería de Educación y Cultura Ed., Murcia, pp. 467-474.

Gómez Vizcaíno, A.; Munuera Navarro, D. (2002). "El sistema defensivo de los Austrias", in Estudio y catalogación de las defensas de Cartagena y su bahía, Comunidad Autónoma de la Región de Murcia, Consejería de Educación y Cultura, Dirección General de Cultura Ed., Murcia, pp. 122-170.

Gómez Vizcaíno, A.; Munuera Navarro, D. (2002). "La fortificación del siglo XVIII", in Estudio y catalogación de las defensas de Cartagena y su bahía, Comunidad Autónoma de la Región de Murcia, Consejería de Educación y Cultura, Dirección General de Cultura Ed., Murcia, pp. 171-237.

Martínez López, J.A.; Iniesta Sanmartín, A. (2002). Estudio y catalogación de las defensas de Cartagena y su bahía, Comunidad Autónoma de la Región de Murcia, Consejería de Educación y Cultura, Dirección General de Cultura Ed., Murcia.

Martínez López, J.A. (2015). "Cartagena ilustrada. La nueva fortificación urbana a través de las colecciones cartográficas de los ingenieros militares", $P+C$ : proyecto y ciudad, 6, pp. 33-50.

Martínez López, J.A., Munuera Navarro, D. (2005). Atlas Político y Militar del Reino de Murcia formado por el Capitán de Infantería e Ingeniero Ordinario de los R. Ejércitos Don Juan José Ordovás. Año 1799 (reedición), MIMARQ Arquitectura y Arqueología Ed., Murcia.

Marzal Martínez, A. (1993). "Cartagena, modelo de Ingeniería militar del siglo XVIII”, in Cartagena 1755 según las Respuestas Generales del Catastro de Ensenada, Centro de Gestión Catastral y Cooperación Tributaria Ed., Madrid, pp. 29-61.

Rubio Paredes, J.M. (1997). "Los castillos de Galeras y Atalaya de Cartagena", Castillos de España. Asociación Española de Amigos de los Castillos, 108, pp. 3-22.

Rubio Paredes, J.M. (2000). Historia de las torres vigías de la costa del reino de Murcia (ss. XVI-XIX), Real Academia Alfonso X El Sabio Ed., Murcia.

Sanjurjo Álvarez, A. (2016). La escalera de caracol en los tratados de cantería españoles de la Edad Moderna y su presencia en el patrimonio construido hispánico: estudio geométrico y constructivo, Tesis Doctoral, Escuela Técnica Superior de Arquitectura de Madrid, Universidad Politécnica de Madrid, Madrid.

Torres Balbás, L. (1960). "Crónica arqueológica de la España musulmana, XLVII", Al-Ándalus: revista de las Escuelas de Estudios Árabes de Madrid y Granada, 25, 2, pp. 419-444. 